\title{
Training Rejection Interpretation in Eating disordeRs (TRIER): Preliminary Findings of a Feasibility Study in Adolescents with Anorexia Nervosa
}

\author{
Valentina Cardi ${ }^{1,6}$ (D) Robert Turton $^{1} \cdot$ Charlie Brazil $^{2,3} \cdot$ Amy Harrison $^{4} \cdot$ Katie Rowlands $^{1} \cdot$ Janet Treasure $^{1}$. \\ Colette Hirsch ${ }^{5}$
}

Published online: 19 July 2019

(c) The Author(s) 2019

\begin{abstract}
This study investigated expectations of social rejection in adolescents with anorexia nervosa. Female adolescents $(N=24)$ admitted for hospital treatment completed both a positive interpretation bias training (experimental training) and a 50\% positive and 50\% negative interpretation bias training (control training), in a counterbalanced order, in two different testing sessions using a within-subjects design. The primary outcome for the training was the frequency of negative interpretations produced in the sentence completion task. At baseline, participants produced more negative than benign interpretations of ambiguous social scenarios (medium/large effect size). Following experimental training, participants produced fewer negative and more benign interpretations compared to control training (medium/large effect sizes). A trend for higher levels of self-esteem following virtual ostracism was found in the experimental condition compared to the control condition (small/ medium effect size). Interpretation bias modification has the potential to improve resilience to rejection in adolescents with anorexia nervosa.
\end{abstract}

Keywords Anorexia nervosa $\cdot$ Interpretation bias $\cdot$ Computerised training $\cdot$ Rejection $\cdot$ Social $\cdot$ Interpersonal

\section{Introduction}

Valentina Cardi and Robert Turton were joint first authors and Janet Treasure and Colette Hirsch were joint last authors.

Valentina Cardi

valentina.cardi@kcl.ac.uk

1 Section of Eating Disorders, Department of Psychological Medicine, Institute of Psychiatry, Psychology and Neuroscience, King's College London, London, UK

2 UCL Great Ormond Street Institute of Child Health, London, UK

3 Ellern Mede Ridgeway, London, UK

4 Department of Psychology and Human Development, Institute of Education, University College London, 25 Woburn Square, London WC1H 0AA, UK

5 Department of Psychology, Institute of Psychiatry, Psychology and Neuroscience, King's College London, London, UK

6 Section of Eating Disorders, Department of Psychological Medicine, Institute of Psychiatry, Psychology and Neuroscience, King's College London, 103 Denmark Hill (Room B3), London SE5 8AF, UK
Adolescence is a time of emerging independence and selfidentity that occurs in the context of heightened sensitivity towards social rejection (Sebastian et al. 2011). The "imbalance" model proposes that this period of life is characterised by intense and frequent negative affect due to differential times of maturation of brain regions that underline emotion perception (i.e. subcortical brain regions) and emotion regulation (cortical circuits) (Casey et al. 2010). In line with the prediction of this model, social rejection during this time is particularly painful because whilst their brain is fully equipped to detect the saliency of the event and experience its emotional intensity, it is not as well equipped to regulate the responses to it (Nelson et al. 2005). The increased vulnerability to an imbalance between affective (e.g. increased anxiety and negative mood) and regulatory systems (e.g. reduced emotion regulation skills) in the salient context of social relationships is likely to underline the dramatic incidence of mental health problems in adolescence (Kessler et al. 2005). 
Anorexia nervosa is a mental health problems often triggered in adolescence and primarily characterised by restriction of food intake and physical deterioration. A lifetime prevalence of $0.3 \%$ for anorexia nervosa has been found in adolescent males and females aged 13 to 18 years (Swanson et al. 2011); however dieting and abnormal eating behaviours are reported in a larger proportion of individuals in population-based studies (e.g. $50 \%$ of females and $25 \%$ of males reporting dieting in the previous year; Neumark-Sztainer et al. 2011). Adverse interpersonal experiences predate the onset of the anorexia nervosa at least in a subgroup of individuals (Cardi et al. 2018a). Negative social experiences include episodes of bullying, criticism from others (especially in relation to weight and shape), exposure to the sudden death of a close relative and poor care and communication in the family, amongst others (Cardi et al. 2018b). These experiences are broadly salient, because of their potential to threaten the universal need of social belonging and could be specifically relevant to individuals with anorexia nervosa, who tend to demonstrate involuntary submissiveness, fear of negative evaluation from others and sensitivity to rejection (Cardi et al. 2013a; Rieger et al. 2010). This is in line with the proposal that one of the functions of abnormal eating behaviours might be to increase social acceptance, silence negative emotions due to interpersonal adversity or gain control in non-social areas (Arcelus et al. 2011; Goss and Allan 2009; Rieger et al. 2010).

We are interested in the hypothesis that increased sensitivity to social threat is a modifiable factor in eating disorders. In a series of studies, we have demonstrated that women with an eating disorder have, on average, an increased tendency to interpret social information in a negative rather than benign way. In particular, they display an attention bias towards rejecting faces and faces expressing social rank-related information compared to neutral faces (Cardi et al. 2013a, 2014) and generate negative interpretations of ambiguous social scenarios that depict the risk of rejection to a greater extent than healthy women (Cardi et al. 2015, 2017). These findings expand the evidence base on cognitive biases towards disorder-specific stimuli in patients with eating disorders (i.e. body and food images; e.g. Brockmeyer et al. 2018; Cardi et al. 2013b) and align to the literature on threat-related biases in patients with anxiety disorders (Hirsch et al. 2006). This is not surprising, given the phenotypic and genetic overlap, and high comorbidity between anxiety disorders and anorexia nervosa (e.g. Duncan et al. 2017). We also provided evidence that attention and interpretation biases to social stimuli are amenable to change through computerised cognitive bias modification training in anorexia nervosa (Cardi et al. 2015; Turton et al. 2018), similarly to what has been demonstrated in anxiety disorders.
Cognitive bias modification paradigms have been developed to modify negative cognitive biases in interpretation and attention. In clinical populations, these paradigms have been found to reduce negative interpretation and attention biases towards depression- and anxiety-specific stimuli with a moderate effect (Hedges' $g=0.49$ ) (Hallion and Ruscio 2011). More recently, a meta-analysis of cognitive bias modification studies for interpretation bias in children and adolescents has shown moderate effects for changing a negative interpretation bias (Hedges' $g=.77$ ) and a small effect size for reducing anxiety levels post training (Hedges' $g=.21$; Krebs et al. 2018).

Despite the evidence of increased sensitivity to social rejection in adolescence and the peak of onset of eating disorder symptoms in this period of life, no studies have assessed the presence of cognitive biases towards social stimuli in adolescents with eating disorders. It follows that the goals of this study are: (1) to test the existence of such biases in adolescents with anorexia nervosa admitted for intensive hospital care, (2) to assess the relationship between cognitive biases and disorder-specific symptoms and (3) to examine the amenability to change through cognitive bias modification procedures. We hypothesised that: (1) adolescent patients with anorexia nervosa would report high expectation of social rejection and produce more negative than benign interpretations of ambiguous social scenarios at baseline, (2) the baseline interpretation bias towards rejection would be associated with eating disorder-specific features (eating disorder symptoms), self-reported expectations of rejection and symptoms and anxiety and depression and (3) a single computerised experimental training session whereby ambiguous scenarios are always followed by benign resolutions (Training Rejection Interpretation in Eating disordeRs-TRIER) would be effective in reducing frequency of negative interpretations and increase frequency of positive interpretations compared to a control training condition (i.e. ambiguous scenarios followed by benign resolutions $50 \%$ of times and $50 \%$ of times followed by negative resolutions). We also hypothesised that the experimental training would be more effective to increase resilience to social exclusion during a virtual ball tossing game (Cyberball game, Williams et al. 2000) compared to the control training condition.

\section{Method}

\section{Participants}

Twenty-four adolescent participants with anorexia nervosa were recruited from a child and adolescent mental health inpatient unit for eating disorders. Inclusion criteria were: females between the ages of 14 and 18 years old, a diagnosis of anorexia nervosa or atypical anorexia nervosa (i.e. all 
diagnostic criteria met except for weight) based upon the DSM-5 criteria (American Psychiatry Association 2013) and fluency in English. Participants were told that the study was aimed at investigating how adolescents think about social scenarios.

All procedures performed were in accordance with the ethical standards of a research ethics committee for studies undertaken within the UK National Health Service (approval reference number: 14/LO/2166) and with the 1964 Helsinki declaration and its later amendments or comparable ethical standards.

\section{Design}

A within-subjects design was used. The two versions of the training were completed on different days with the two sessions arranged a week apart. The order of the conditions was counterbalanced using the random number generator function in Microsoft Excel ${ }^{\circledR}$. This resulted in $50 \%$ of the participants receiving the experimental condition and 50\% the control condition in the first session. The choice of a within-subjects design follows the ethical consideration of ensuring all participants would receive both versions of the training. This design was chosen also to reduce the variance due to individual differences in cognitive processing and therefore to improve statistical power.

\section{Materials}

\section{Eating Disorders Examination Questionnaire (EDE-Q; Fairburn and Beglin 1994)}

This measure assesses eating disorder-related symptoms over the past 28 days. Participants rate frequency of symptoms on a seven-point Likert scale ranging from 0 (e.g. "no days") to 6 (e.g. 1"every day"). Higher scores correspond to higher levels of symptoms. It consists of four subscales (i.e., eating concern, shape concern, weight concern and dietary restraint) and a global scale. The mean global score for eating disorder patients was $3.09(\mathrm{SD}=0.83)$ compared to 1.30 $(\mathrm{SD}=0.96)$ in healthy controls, in a paper which validated this scale to screen for eating disorders in community samples (Mond et al. 2004). In the same paper, the EDE-Q was found to have good internal consistency, convergent validity and test-retest reliability (Mond et al. 2004). The Cronbach's alpha for the total score in this study was 0.88 .

\section{Revised Children's Anxiety and Depression Scale (RCADS; Chorpita et al. 2000)}

This 47-item questionnaire assesses symptoms of anxiety and depression. Items are rated on a 4-point scale, ranging from 0 ("never") to 3 ("always"). The RCADS is a valid and reliable questionnaire for multi-ethnic late childhood populations (Chorpita et al. 2000). The Cronbach's alphas in this study suggested excellent internal consistency for the anxiety scale (0.95) and good internal consistence for the depression scale (0.84).

\section{Children's Rejection Sensitivity Questionnaire (CRSQ; Downey et al. 1998)}

This measure presents 12 peer-related vignettes depicting situations where there is a possibility of rejection. For each vignette, participants must first rate how anxious they would be in that situation, using a six-point scale ranging from 1 (not nervous) to 6 (very nervous). Participants are then asked to specify how likely it is that the other person would respond with rejection or acceptance, choosing between three categories (i.e., "yes", "no" or "maybe"). Research shows good internal consistency for this measure (Downey et al. 1998). The Cronbach's alpha in this study was 0.94 .

\section{Visual Analogue Scales (VASs)}

Visual analogue scales (i.e., $10 \mathrm{~cm}$ long) were used to determine participants' levels of anxiety (anchored by 'not at all' and 'extremely') and mood (anchored by 'extremely low' and 'extremely high').

Sentence Completion Task (Adapted from Cardi et al. 2015; Hayes et al. 2010; Huppert et al. 2007)

In this computer-based task, participants listen to 12-stem sentences (i.e., two practice trials and 10 test trials) that describe socially ambiguous scenarios that involve the risk of rejection (e.g., "Your teacher calls you into her office to tell you that you did something...”). Participants write down as many short word completions to the scenarios as they can and are then asked to indicate with an asterisk the completion that they think 'best' completes the scenario. In this study, the scenarios were adapted from those used by Cardi et al. (2015) in a population of adult patients with anorexia nervosa, so that they were age appropriate (e.g., relating to school as opposed to work). Two raters (R.T. and J.L.) who were blind to the conditions of the sample, scored participants' 'best' completions as negative or benign. The interrater reliability was checked using the kappa statistic (.91), and a third party rater resolved any disagreements (V.C.).

\section{Interpretation Bias Training (Modified from Cardi et al.} 2015)

This included a total of 100 trials: 90 interpretation modification trials and an additional 10 test trials presented in a random order. Scenarios were presented to participants over 
headphones and each described a situation that was ambiguous with regards to the risk of social rejection, until the last word resolved the ambiguity in either a benign or negative manner. For example: "You text your sister to arrange going shopping but she doesn't reply. You think she is (really busy/ ignoring you)". At the conclusion of each scenario, a comprehension question was given with a yes/no option (e.g., "Is your sister too busy to reply right now?"), and the correct answer confirmed the outcome of the scenario. The aim of this question was to reinforce the valence of the modification trial. A computer-generated tone followed the yes/no response to give feedback on whether the answer was correct or incorrect. Test trials were also included. These trials kept their ambiguity and were followed by an open question (e.g., "Are you concerned that your sister is ignoring you?"). The response to the questions in the test trials were taken as an indication of the type of interpretation (i.e. benign or negative) spontaneously generated by participants. The experimental condition (TRIER) involved entirely benign endings to the social scenarios, and the control condition included a 50:50 split of benign/negative endings.

The experimental and control versions of the trainings were administered in five blocks each. Each block contained 18 training scenarios and two test trials. Participants were offered an opportunity to take a break in between each block. To increase the ecological validity of the training, the scenarios originally used in an adult sample of patients with anorexia nervosa (Cardi et al. 2015) were shared with five adolescent patients receiving treatment on the ward where the final study was conducted. The researcher (AH) and the five patients went through each scenario and discussed the wording and relevance to the adolescent age group. Where the adolescents indicated that the wording and/or scenario were not relevant, this was amended based on their feedback.

\section{Filler Task: The Speed of Comprehension Test (Baddeley et al. 1992)}

Following the training, participants completed a filler task to eliminate any potential carry over effects of the training on participants' mood (Hayes et al. 2010). The task consisted of 100 sentences, half of which were "true" and half of which were "false", (e.g., "Nuns live in trees"). Participants were required to indicate with a tick which sentences were true.

\section{Cyberball Task (Williams et al. 2000)}

This computer-based task is a ball throwing game between the participant and two other virtual players. In this study, there were a total of 30 throws between the players, with participants being thrown the ball only six times, randomly during the game. After playing the game, participants were asked to complete the Needs Threat Scale (van Beest and
Williams 2006) to measure their feelings of self-esteem, belonging, control and meaningful existence on a 7-point scale. These questions were piloted with a small group of healthy adolescents to ensure they were age-appropriate. The Cronbach's alphas were 0.85 for self-esteem, 0.72 for belonging, 0.44 for control and 0.78 for meaningful existence.

\section{Procedure}

Informed consent was obtained from all participants included in the study. Consent was gained from both the participants and their guardians for patients younger than 16 . The study procedure involved completing the sentence completion task, which was done on a laptop with headphones (using E-PRIME, version two; E-Prime 2016). Interpretation training was then administered, followed by the second sentence completion task. The visual analogue scales to measure anxiety and mood were also completed before and after the training. A filler task was then given, and the online Cyberball game was completed. The first and second testing sessions were conducted approximately 1 week apart. Participants were fully debriefed at the end of the second study session. Please see Fig. 1 for an overview of the study procedure.

\section{Data Analyses}

Clinical characteristics were examined using descriptive statistics (SPSS, version 22). Wilcoxon-signed ranks tests were calculated for data that were not normally distributed.

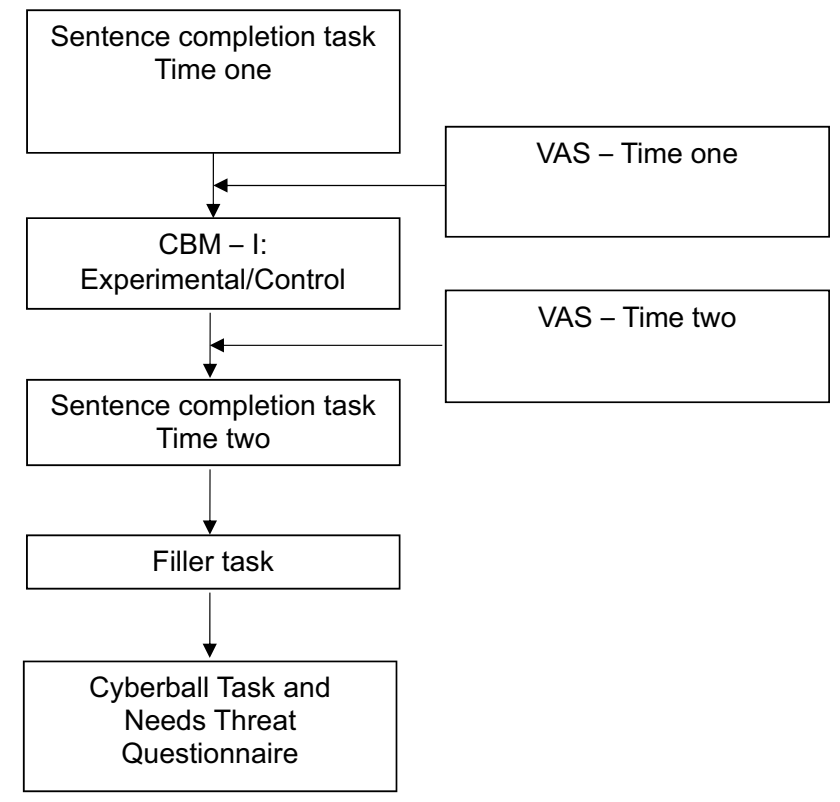

Fig. 1 Flow-chart outlining the procedure of the study 
In particular, they were used to compare: (1) frequencies of negative and benign 'best' interpretations the first time that participants completed the sentence completion task (regardless whether this was in the context of the experimental or control condition) to examine interpretation bias for social stimuli at baseline, (2) frequencies of negative and benign "best" interpretations before and after each study condition to assess training effects, (3) frequencies of negative and benign interpretations of test trials and (4) scores on the Needs Threat Scale following the Cyberball task between the experimental and control conditions. Spearman's rank correlations were used to establish the associations between interpretation bias at baseline and eating disorder-specific clinical characteristics. Participant's responses on the visual analogue scales for anxiety and mood were normally distributed and therefore analysed using separate mixed effects linear models for a $2 \times 2$ design (i.e., Training condition $\times$ Time). This analysis was completed on Stata version $14^{\circledR}$ (StataCorp 2015) and was bootstrapped at 10,000 repetitions.

A $p$ value of $<.05$ was used as the threshold for statistical significance. Correlation coefficients were used to establish effect sizes for non-normally distributed data $(r=0.1$, small; $r=0.3$, medium; $r=0.5$, large).

\section{Results}

\section{Demographic and Clinical Characteristics}

Table 1 shows the sample's demographic and clinical characteristics. Participants' mean age was 15.87. At the time of their participation in the study, participants had been admitted to hospital for 116.75 days on average and had a mean

Table 1 Means, standard deviations and minimum and maximum values of the sample's demographics and clinical characteristics. EDEQ Eating Disorders Examination Questionnaire, RCADS Revised Children's Anxiety and Depression Scale; CRSQ Children's Rejection Sensitivity Questionnaire

\begin{tabular}{lll}
\hline Clinical characteristics & Mean (SD) & Min-max \\
\hline Age & 15.87 & $14-17$ \\
Weight for height & $84.17(11.85)$ & $59.82-101.98$ \\
Length of admission (days) & $116.75(116.84)$ & $7-447$ \\
Illness duration (years) & $2.66(1.81)$ & $.0-6$ \\
EDEQ—-weight & $4.18(1.74)$ & $1-6$ \\
EDEQ-shape & $4.76(1.58)$ & $.75-6$ \\
EDEQ-restraint & $3.43(1.86)$ & $0-6$ \\
EDEQ-eating concern & $3(1.63)$ & $.4-6$ \\
EDEQ—-total & $3.75(1.54)$ & $.59-6$ \\
RCADS-total anxiety & $57.26(22.59)$ & $18-92$ \\
RCADS-major depression & $16.74(6.48)$ & $4-27$ \\
CRSQ & $18.81(7.78)$ & $5.67-32.5$ \\
\hline
\end{tabular}

duration of illness of 2.66 years. Sixty-seven percent of the sample was taking psychotropic medication at the time of their participation in the study. Medications prescribed included Fluoxetine, Sertraline, Aripiprazole, Lamotragine, Olanzapine and Mirtazapine. A co-morbid diagnosis of anxiety or mood disorder was reported by clinicians for $50 \%$ of the participants.

Participants' scores on the depression and anxiety scales of the Revised Children's Anxiety and Depression Scale were higher than those of an age-matched control sample of female students tested in a previous study, although statistical comparisons between these two sets of data could not be performed (Chorpita et al. 2000). Similarly, participants scored higher than a control sample of adolescents on expectations of rejection (i.e. scores ranging between 0 and 36; mean of control sample of adolescents $=8.16, S D=3.91$; Downey et al. 1998).

\section{Baseline Negative and Benign Interpretations}

At baseline, participants produced significantly more negative 'best' interpretations $(M d n=7, I Q R=6-9)$ than benign 'best' interpretations $(M d n=3, I Q R=1-4)$ on the sentence completion task $(Z=3.91, p<.01, r=.56$, large effect size $)$ (Fig. 2). The negative interpretation bias for social stimuli significantly correlated with expectation of rejection $\left(r_{s}=.49, p=.03\right)$ and with symptoms of anxiety $\left(r_{s}=.69\right.$, $p<.01)$ and depression $\left(r_{s}=.42, p=.04\right)$. There was no significant correlation with EDE-Q total score $\left(r_{s}=.3, p=.23\right)$. These findings indicate that adolescent patients have a negative interpretation bias towards ambiguous scenarios and that this bias correlates with anxiety, depression and expectation of rejection.

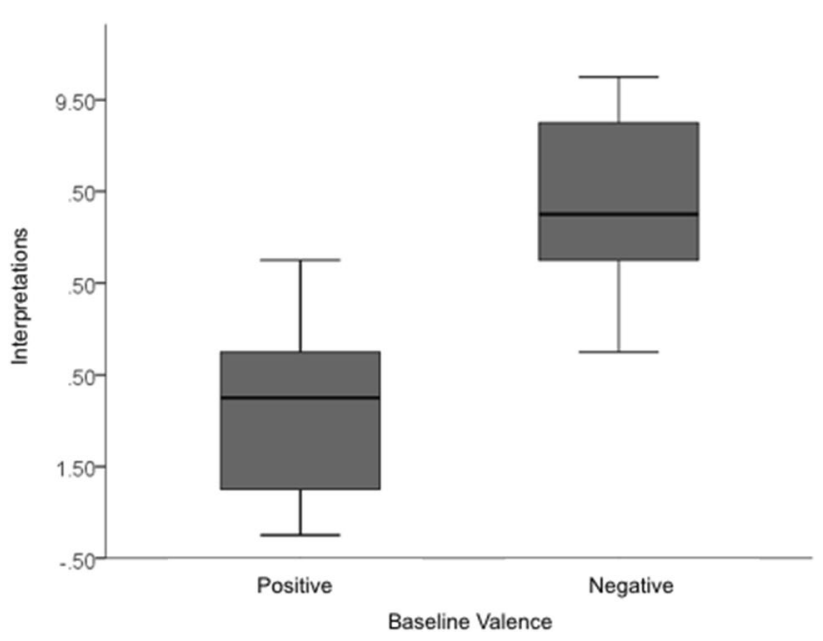

Fig. 2 Medians and inter-quartile ranges of positive and negative interpretations produced at baseline by adolescents with anorexia nervosa 


\section{Interpretation Bias Training}

\section{Accuracy}

Participants' accuracy levels on the comprehension trials did not significantly differ between the experimental $(M d n=76, I Q R=60-84.75)$ and control $(M d n=77.5$, $I Q R=61.75-83.5 ; Z=-.7, p=.48, r=.1$, small effect size) conditions.

\section{Interpretation of Test Trials}

Participants made significantly more benign responses to the test trials in the experimental $(M d n=6.5, I Q R=3.25-7.75)$ compared to the control condition $(M d n=4, I Q R=2-5$; $Z=3.39, p=.01, r=.49$; large effect size; Fig. 3 ). These data indicate that the experimental condition was superior in producing positive interpretations of the test trials.

\section{Outcomes of Interpretation Bias Training}

\section{Frequency of Negative and Benign Interpretations}

Following the experimental training (positive training stimuli), participants made significantly fewer negative interpretations post-training $(M d n=6, I Q R=4.25-8.75)$ compared to pre-training $(M d n=8, I Q R=5.25-9 ; Z=-2.92$, $p<.01, r=.42$, medium effect size). They also made significantly more benign interpretations post-training $(M d n=4$, $I Q R=1.25-5.75)$ than pre-training $(M d n=2, I Q R=1-3.75$; $Z=-3.15, p<.01, r=.45$, medium/large effect size).

In the control condition (50\% positive and $50 \%$ negative training stimuli), there were no significant differences

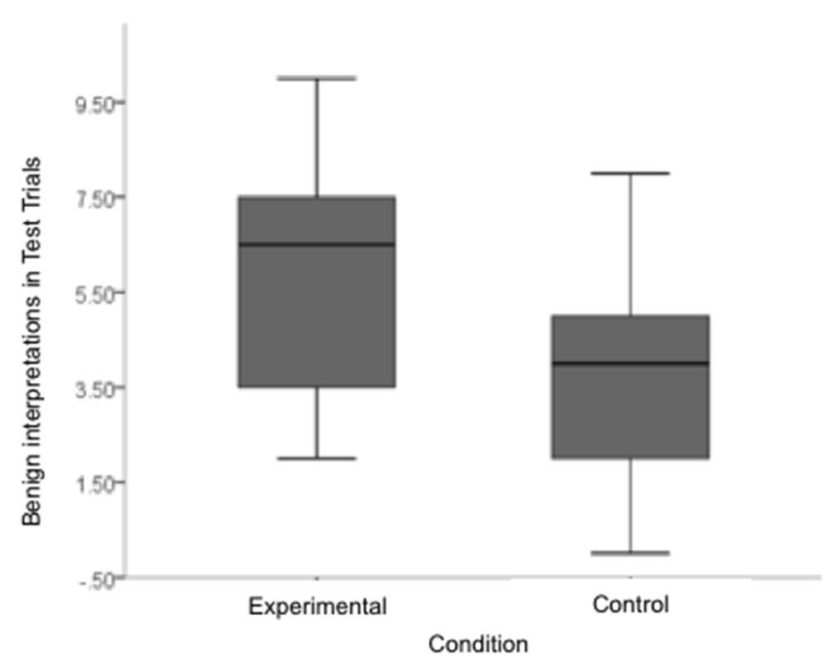

Fig. 3 Medians and inter-quartile ranges of benign interpretations produced in the experimental and control conditions by adolescents with anorexia nervosa for negative (Pre $M d n=6, I Q R=6-8$; Post $M d n=6.5$, $I Q R=4.25-9 ; Z=-.40, p=.68, r=.06$, negligible effect size) or benign interpretations (Pre $M d n=4, I Q R=2-4$; Post $M d n=2.5, I Q R=1-5 ; Z=-.33, p=.74, r=.05$, negligible effect size) between pre- and post-training. These findings indicate that training effects only occurred in the experimental condition.

\section{Mood and Anxiety Ratings}

The $2 \times 2$ mixed effects linear models (i.e., Training Condition $\times$ Time) for mood and anxiety ratings showed that there were no significant main effects of Training Condition, Time or significant interaction effects between Training Condition and Time for these two variables.

\section{Responses to Rejection from Others (Cyberball Task)}

There was a trend for participants to report higher levels of self-esteem after the Cyberball task in the experimental $(M d n=13, I Q R=10-15)$ compared to the control condition $(M d n=11, I Q R=8.25-14.5 ; Z=-1.69, p=.09, r=.24$, small/moderate effect size). There were no significant differences between conditions in levels of meaningful existence ( $p=.27, r=.16$, small effect size), feeling of belonging ( $p=.45, r=.11$, small effect size) or feeling of control ( $p=.75, r=.05$, negligible effect size). These findings indicate that the experimental training was associated with a trend-level increase in self-esteem and with no other changes compared to control training.

\section{Discussion}

This study investigated whether adolescents with anorexia nervosa admitted for inpatient care have high levels of expectation of rejection from others and a negative interpretation bias of ambiguous social situations. Furthermore, the frequency of negative and benign interpretations produced before and after computerised interpretation bias training and resilience to rejection from virtual players were compared in the experimental (positive training) and control (50\% positive and $50 \%$ negative training) conditions. As hypothesised, participants reported higher levels of anxious expectation of rejection compared to a normative sample of adolescents. They also produced significantly more negative interpretations of ambiguous social scenarios than benign interpretations. The frequency of negative interpretation was associated with self-reported expectation of rejection from others and symptoms of anxiety and depression.

Training effects were found only in the positive training condition, as demonstrated by participants producing significantly more benign interpretations and significantly 
fewer negative interpretations post-training compared to pretraining. No changes in frequencies of negative or benign interpretations were found in the control version of the training. In the experimental condition (positive training), participants also produced more positive interpretations of test trials, compared to the control condition. No significant differences in the behavioural responses to virtual ostracism induced by the Cyberball task were found between the experimental and control trainings, except for a trend level improvement in self-esteem in the experimental condition.

Overall, these findings align to the emerging literature on cognitive biases to rejection in eating disorders, which demonstrate that patients have increased expectancy of rejection from others and a negative attention and interpretation bias to social stimuli (Cardi et al. 2013a, b, 2015, 2017). This study demonstrates that expectations of rejection and negative interpretation bias are relevant also in adolescents suffering from anorexia nervosa.

The finding that a single session of interpretation training can reduce the frequency of negative interpretations and increase the frequency of positive interpretations is consistent with previous studies, mostly conducted with healthy/unselected samples of adolescents (Krebs et al. 2018) and more recently with adolescents diagnosed with major depression disorder (LeMoult et al. 2018). Also, in line with these studies, is the finding that effects on selfreported measures (i.e. self- but not assessor-rated negative intrusions; Krebs et al. 2018 and self-esteem in our study) appear weaker. Given the illness severity of the participants included within this study, it could be the case that multiple training sessions are needed to produce a larger effect on ecologically-valid measures of social functioning and specific eating disorder symptoms.

A strength of this study is the adaptation of the interpretation training materials specifically for adolescents with anorexia nervosa. This follows the recommendation that research on interpretation bias training should ensure that training materials are both relevant and realistic for the participant group (Hirsch et al. 2016; Hughes et al. 2016). There are potential limitations to the study. The relatively small sample size might limit the power to detect changes in self-reported measures and the use of a single training session, as opposed to multiple training sessions, could reduce training's efficacy (e.g. Heitmann et al. 2018; Hirsch et al. 2018). The use of a within-subject design might confound findings if participants become aware of the differences between conditions. In this study, participants were not asked to provide a guess as to which training condition they had completed at the end of each session and therefore the occurrence of potential demand effects cannot be excluded. The Cyberball task did not have an "inclusion block" (block in which the participant is thrown the ball an equal number of times than the other two players) before the "exclusion block" (Masten et al. 2011), which could reduce participants' focus on exclusion. Also, this task was only administered after training and therefore differences in self-esteem between training conditions could be confounded by baseline, pre-existing differences in self-esteem (e.g. at baseline, participants in the experimental training condition might have higher levels of self-esteem compared to participants in the control condition).

\section{Clinical Implications}

No participants requested to interrupt the study and accuracy levels in both training conditions were over $80 \%$. This suggests that the training was feasible for patients in our study. However, considering the advantages of using multiple training sessions (Heitmann et al. 2018; Hirsch et al. 2018), future research should examine the use of gamified strategies and technology, such as virtual reality (Otkhmezuri et al. 2019), to ensure engagement with the training over time. Finally, it would be important to assess whether changes in underlying cognitive biases are associated with changes in social behaviour also, such as greater social connectedness and quality of social life.

\section{Conclusions}

To date, this is the first study to use interpretation bias training for social rejection in adolescents with anorexia nervosa. A single session of positive training significantly reduced a negative interpretation bias for social stimuli and facilitated a more benign interpretational style in comparison to a control condition (50\% positive $/ 50 \%$ negative). There was also a trend for the adolescents to have greater resilience in response to a social rejection task.

Acknowledgements The authors thank all of the patients and healthcare assistants at Ellern Mede who contributed to and supported the data collection. Thank you to Dr Jenni Leppanen for her assistance with the scoring of the interpretation bias data.

Funding To conduct this work, R.T. was funded by the Medical Research Council (MRC) and the Psychiatry Research Trust (PRT) (Grant PCPTAAR). J.T. and C.H. receive salary support from the National Institute for Health Research (NIHR), Mental Health Biomedical Research Centre at South London and Maudsley NHS Foundation Trust and King's College London. V.C. is funded by the National Institute for Health Research and receives salary support from the Mental Health Biomedical Research Centre at South London and Maudsley NHS Foundation Trust. A.H. is supported by a grant from the Medical Research Council (MR/S019707/1). The views expressed in this article are those of the author(s) and not necessarily those of King's College London, the NIHR, MRC, PRT, the or the Department of Health. 


\section{Compliance with Ethical Standards}

Conflict of Interest Valentina Cardi, Robert Turton, Charlie Brazil, Amy Harrison, Katie Rowlands, Janet Treasure, Colette Hirsch declare that they have no conflict of interest.

Informed Consent Informed consent was obtained from all individual subjects who participated in the study.

Animal Rights No animal studies were carried out by the authors for this article.

Open Access This article is distributed under the terms of the Creative Commons Attribution 4.0 International License (http://creativeco mmons.org/licenses/by/4.0/), which permits unrestricted use, distribution, and reproduction in any medium, provided you give appropriate credit to the original author(s) and the source, provide a link to the Creative Commons license, and indicate if changes were made.

\section{References}

American Psychiatric Association. (2013). Diagnostic and statistical manual of mental disorders. Arlington: American Psychiatric Publishing.

Arcelus, J., Mitchell, A. J., Wales, J., \& Nielsen, S. (2011). Mortality rates in patients with anorexia nervosa and other eating disorders. A meta-analysis of 36 studies. Archives of General Psychiatry, 68(7), 724-731.

Baddeley, A., Emslie, H., \& Nimmo-Smith, I. (1992). The speed of comprehension test: Version A. In speed and capacity of language processing test. Suffolk: Thames Valley Test Company.

Brockmeyer, T., Anderle, A., Schmidt, H., Febry, S., Wünsch-Leiteritz, W., Leiteritz, A., et al. (2018). Body image related negative interpretation bias in anorexia nervosa. Behaviour Research and Therapy, 104, 69-73.

Cardi, V., Di Matteo, R., Corfield, F., \& Treasure, J. (2013a). Social reward and rejection sensitivity in eating disorders: An investigation of attentional bias and early experiences. World Journal of Biological Psychiatry, 14(8), 622-633.

Cardi, V., Di Matteo, R., Gilbert, P., \& Treasure, J. (2014). Rank perception and self-evaluation in eating disorders. International Journal of Eating Disorders, 47(5), 543-552.

Cardi, V., Esposito, M., Bird, G., Rhind, C., Yiend, J., Schifano, S., et al. (2015). A preliminary investigation of a novel training to target cognitive biases towards negative social stimuli in Anorexia Nervosa. Journal of Affective Disorders, 188, 188-193.

Cardi, V., Lounes, N., Kan, C., \& Treasure, J. (2013b). Meal support using mobile technology in Anorexia Nervosa. Contextual differences between inpatient and outpatient settings. Appetite, 60(1), 33-39.

Cardi, V., Mallorqui-Bague, N., Albano, G., Monteleone, A. M., Fernandez-Aranda, F., \& Treasure, J. (2018a). Social difficulties as risk and maintaining factors in anorexia nervosa: A mixed-method investigation. Frontiers in Psychiatry, 9, 12.

Cardi, V., Tchanturia, K., \& Treasure, J. (2018b). Premorbid and illness-related social difficulties in eating disorders: An overview of the literature and treatment developments. Current Neuropharmacology, 16(8), 1122-1130.

Cardi, V., Turton, R., Schifano, S., Leppanen, J., Hirsch, C. R., \& Treasure, J. (2017). Biased interpretation of ambiguous social scenarios in anorexia nervosa. European Eating Disorders Review, 25(1), 60-64.
Casey, B. J., Jones, R. M., Levita, L., Libby, V., Pattwell, S., Ruberry, E., et al. (2010). The storm and stress of adolescence: Insights from human imaging and mouse genetics. Developmental Psychobiology, 52(3), 225-235.

Chorpita, B. F., Yim, L., Moffitt, C., Umemoto, L. A., \& Francis, S. E. (2000). Assessment of symptoms of DSM-IV anxiety and depression in children: A revised child anxiety and depression scale. Behavior Research and Therapy, 38(8), 835-855.

Downey, G., Lebolt, A., Rincon, C., \& Freitas, A. L. (1998). Rejection sensitivity and children's interpersonal difficulties. Child Development, 69(4), 1074-1091.

Duncan, L., Yilmaz, Z., Gaspar, H., Walters, R., Goldstein, J., Anttila, V., et al. (2017). Significant locus and metabolic genetic correlations revealed in genome-wide association study of anorexia nervosa. The American Journal of Psychiatry, 174(9), $850-858$.

Fairburn, C. G., \& Beglin, S. J. (1994). Assessment of eating disorders: Interview or self-report questionnaire? International Journal of Eating Disorders, 16(4), 363-370.

Goss, K., \& Allan, S. (2009). Shame, pride and eating disorders. Clinical Psychology and Psychotherapy, 16(4), 303-316.

Hallion, L. S., \& Ruscio, A. M. (2011). A meta-analysis of the effect of cognitive bias modification on anxiety and depression. Psychological Bulletin, 137(6), 940-958.

Hayes, S., Hirsch, C. R., Krebs, G., \& Mathews, A. (2010). The effects of modifying interpretation bias on worry in generalized anxiety disorder. Behavior Research and Therapy, 48(3), 171-178.

Heitmann, J., Bennik, E. C., van Hemel-Ruiter, M. E., \& de Jong, P. J. (2018). The effectiveness of attentional bias modification for substance use disorder symptoms in adults: A systematic review. Systematic Reviews, 7, 160.

Hirsch, C. R., Clark, M., \& Mathews, A. (2006). Imagery and interpretation in social phobia: Support for the combined cognitive biases hypothesis. Behavior Therapy, 37(3), 223-236.

Hirsch, C. R., Krahé, C., Whyte, J., Loizou, S., Bridge, L., Norton, S., et al. (2018). Interpretation training to target repetitive negative thinking in Generalized Anxiety Disorder and Depression. Journal of Consulting and Clinical Psychology, 86(12), 1017-1030.

Hirsch, C. R., Meeten, F., Krahe, C., \& Reeder, C. (2016). Resolving ambiguity in emotional disorders: The nature and role of interpretation biases. Annual Review of Clinical Psychology, 12, 281-305.

Hughes, A. M., Gordon, R., Chalder, T., Hirsch, C. R., \& Moss-Morris, R. (2016). Maximizing potential impact of experimental research into cognitive processes in health psychology: A systematic approach to material development. Britith Journal of Health Psychology, 21(4), 764-780.

Huppert, J. D., Pasupuleti, R. V., Foa, E. B., \& Mathews, A. (2007). Interpretation biases in social anxiety: Response generation, response selection, and self-appraisals. Behavor Research and Therapy, 45(7), 1505-1515.

Kessler, R. C., Berglund, P., Demler, O., Jin, R., Merikangas, K. R., \& Walters, E. E. (2005). Lifetime prevalence and age-of-onset distributions of DSM-IV disorders in the National Comorbidity Survey Replication. Archives of General Psychiatry, 62(6), 593-602.

Krebs, G., Pile, V., Grant, S., Degli Esposti, M., Montgomery, P., \& Lau, J. Y. F. (2018). Research review: Cognitive bias modification of interpretations in youth and its effect on anxiety: A meta-analysis. Journal of Child Psychology and Psychiatry, 59(8), 831-844.

LeMoult, J., Colich, N., Joormann, J., Singh, M. K., Eggleston, C., \& Gotlib, I. H. (2018). Interpretation bias training in depressed adolescents: Near- and far-transfer effects. Journal of Abnormal Child Psychology, 46(1), 159-167.

Masten, C. L., Eisenberger, N. I., Borofsky, L. A., McNealy, K., Pfeifer, J. H., \& Dapretto, M. (2011). Subgenual anterior cingulate responses to peer rejection: A marker of adolescents' risk for depression. Development and Psychopathology, 23(1), 283-292. 
Mond, J. M., Hay, P. J., Rodgers, B., Owen, C., \& Beumont, P. J. (2004). Validity of the Eating Disorder Examination Questionnaire (EDE-Q) in screening for eating disorders in community samples. Behavior Research and Therapy, 42(5), 551-567.

Nelson, E. E., Leibenluft, E., McClure, E. B., \& Pine, D. S. (2005). The social re-orientation of adolescence: A neuroscience perspective on the process and its relation to psychopathology. Psychological Medicine, 35(2), 163-174.

Neumark-Sztainer, D., Wall, M., Larson, N. I., Eisenberg, M. E., \& Loth, K. (2011). Dieting and disordered eating behaviors from adolescence to young adulthood: Findings from a 10-year longitudinal study. Journal of the American Dietetic Association, 111(7), 1004-1011.

Otkhmezuri, B., Boffo, M., Siriaraya, P., Matsangidou, M., Wiers, R. W., Mackintosh, B., ... \& Salemink, E. (2019). Believing is seeing: A proof-of-concept semiexperimental study on using mobile virtual reality to boost the effects of interpretation bias modification for anxiety. JMIR Mental Health, 6(2), e11517.

Rieger, E., Van Buren, D. J., Bishop, M., Tanofsky-Kraff, M., Welch, R., \& Wilfley, D. E. (2010). An eating disorder-specific model of interpersonal psychotherapy (IPT-ED): Causal pathways and treatment implications. Clinical Psychology Review, 30(4), 400-410.

Sebastian, C. L., Tan, G. C., Roiser, J. P., Viding, E., Dumontheil, I., \& Blakemore, S. J. (2011). Developmental influences on theneural bases of responses to social rejection: Implications of social neuroscience for education. Neuroimage, 57(3), 686-694.

Swanson, S. A., Crow, S. J., LeGrange, D., Swendsen, J., \& Merikangas, K. R. (2011). Prevelance and correlates of eating disorders in adolescents. Results from the national comorbidity survery replication adolescent supplement. Archives of General Psychiatry, 68(7), 714-723

Turton, R., Cardi, V., Treasure, J., \& Hirsch, C. R. (2018). Modifying a negative interpretation bias for ambiguous social scenarios that depict the risk of rejection in women with anorexia nervosa. Journal of Affective Disorders, 227, 705-712.

van Beest, I., \& Williams, K. D. (2006). When inclusion costs and ostracism pays, ostracism still hurts. Journal of Personality and Social Psychology, 91(5), 918-928.

Williams, K. D., Cheung, C. K., \& Choi, W. (2000). Cyberostracism: Effects of being ignored over the Internet. Journal of Personality and Social Psychology, 79(5), 748-762.

Publisher's Note Springer Nature remains neutral with regard to jurisdictional claims in published maps and institutional affiliations. 